\title{
In vivo administration of a lentiviral vaccine targets DCs and induces efficient $\mathrm{CDB}^{+} \mathrm{T}$ cell responses
}

\author{
Christoph Esslinger, ${ }^{1}$ Laurence Chapatte, ${ }^{1}$ Daniela Finke, ${ }^{2}$ Isabelle Miconnet, ${ }^{2}$ \\ Philippe Guillaume, ${ }^{1}$ Frédéric Lévy, ${ }^{1}$ and H. Robson MacDonald ${ }^{1}$ \\ ${ }^{1}$ Ludwig Institute for Cancer Research, Lausanne Branch, University of Lausanne, Epalinges, Switzerland \\ ${ }^{2}$ Institut Suisse de Recherche Experimental sur le Cancer, Epalinges, Switzerland
}

\begin{abstract}
The present study evaluates the potential of third-generation lentivirus vectors with respect to their use as in vivo-administered $T$ cell vaccines. We demonstrate that lentivector injection into the footpad of mice transduces DCs that appear in the draining lymph node and in the spleen. In addition, a lentivector vaccine bearing a $\mathrm{T}$ cell antigen induced very strong systemic antigen-specific cytotoxic $T$ lymphocyte $(\mathrm{CTL})$ responses in mice. Comparative vaccination performed in two different antigen models demonstrated that in vivo administration of lentivector was superior to transfer of transduced DCs or peptide/adjuvant vaccination in terms of both amplitude and longevity of the CTL response. Our data suggest that a decisive factor for efficient $\mathrm{T}$ cell priming by lentivector might be the targeting of DCs in situ and their subsequent migration to secondary lymphoid organs. The combination of performance, ease of application, and absence of pre-existing immunity in humans make lentivector-based vaccines an attractive candidate for cancer immunotherapy.
\end{abstract}

J. Clin. Invest. 111:1673-1681 (2003). doi:10.1172/JCI200317098.

\section{Introduction}

The prospects of an antitumor vaccine depend on its ability to induce robust and sustained tumorspecific $T$ cell responses. Since the initiation of such responses is crucially dependent on the presentation of the antigen by DCs, current tumor vaccines are designed to target DCs ex vivo. One approach consists of pulsing DCs in vitro with antigenic peptides before transferring them back to the patient (1-3). In an analogous approach, DCs are transduced with an antigen-recombinant viral vector $(4,5)$. Transduction is thought to result in better antigen processing and more sustained antigen presentation than pulsing $(4$, 6). However, the generation and ex vivo manipulation of DCs is laborious and costly. Therefore, a cell-free vaccine that could be easily administered yet would retain the efficacy of the DC-based approach would be a significant improvement. However, as the majority of recombinant viral vectors are derived from vaccines originally designed to prevent viral infections, pre-existing immunity to the parental virus might

Received for publication October 10, 2002, and accepted in revised form April 1, 2003.

Address correspondence to: Christoph Esslinger, Ludwig Institute for Cancer Research, Chemin des Boveresses 155, CH-1066 Epalinges, Switzerland. Phone: 41-21-692-5985; Fax: 41-21-653-4474; E-mail: christoph.esslinger@isrec.unil.ch. Isabelle Miconnet's present address is: INSERM 487, Institut Gustave Roussy, Villejuif, France.

Conflict of interest: The authors have declared that no conflict of interest exists.

Nonstandard abbreviations used: oligodeoxynucleotide (ODN); cytotoxic T lymphocyte (CTL); 1,4-diazabicyclo-(2.2.2)-octane (DABCO); peripheral blood lymphocyte (PBL); bone

marrow-derived DC (BMDC); expression-forming unit (EFU); incomplete Freund's adjuvant (IFA). interfere with their ability to induce potent antitumor immune responses (7).

In this context, the advent of new, efficient gene delivery vehicles may provide a solution. We and others recently introduced lentiviral vectors (lentivectors) as tools for the ex vivo transduction of DCs and the induction of $\mathrm{T}$ cell responses (8-11). These third generation lentivectors are designed for in vivo gene therapy and to our knowledge exhibit the most advanced safety features so far included in any viral vector system (12) and thus may be suitable for in vivo administration as a vaccine.

This study characterizes the properties of an in vivo administered lentivector-based vaccine with regard to its cellular targets and its ability to induce antigenspecific $\mathrm{CD}^{+} \mathrm{T}$ cell responses. We observed that lentivector administration transduced DCs that were found in the draining lymph node and in the spleen. In addition, the vaccine induced potent $\mathrm{T}$ cell responses as demonstrated by up to $40 \%$ antigen-specific cells among the $\mathrm{CD}^{+}$subset and high levels of specific cytotoxicity.

Comparative vaccination with transduced DCs demonstrated that in vivo administration of lentivector induces equally strong antigen-specific $\mathrm{T}$ cell responses. Moreover, when compared with another cellfree approach, administration of antigenic peptides in combination with $\mathrm{CpG}$ oligodeoxynucleotides (ODNs) emulsified in adjuvant, lentivector induced a 3- to 10fold higher amplitude of response.

\section{Methods}

Mice. B6D2F1 mice (Harlan, Zeist, the Netherlands) were used for $\mathrm{Cw} 3$ vaccination. HLA-Cw3 transgenic mice were used as source of antigen-transgenic DCs (13). 
HLA-A*0201/ $\mathrm{K}^{\mathrm{b}}$ transgenic mice were used for vaccinations with Melan-A/ELA $26-35$ (14). These mice were originally provided by Harlan Sprague-Dawley (Indianapolis, Indiana, USA). Mouse experiments were carried out with permission of the "Service Vétérinaire" of the Canton de Vaud, Swiss Confederation.

Lentivector vaccines. The HLA-Cw3 cDNA was cloned by RT-PCR from P 815 cells stable-transfected with HLA-Cw3 (15) and inserted into lentiviral transfer vector pIRES-EGFPpRRLsin18.PPT.CMV. A minigene encoding the $\mathrm{H}-2 \mathrm{~K}^{\mathrm{d}}$-restricted cytotoxic $\mathrm{T}$ lymphocyte (CTL) antigen of HLA-Cw3 (Cw3 ${ }_{194-203)}$ (15-17) was inserted in pIRES-EGFPpRRLsin 18.PPT.CMV (8). Likewise, a minigene encoding the immunodominant peptide analogue of the HLA-A*0201-restricted Melan-A CTL antigen (ELA E6-35 ) was cloned in pIRESEGFPpRRLsin18.PPT.CMV. The mutation at position 27 (Ala to Leu) results in increased immunogenicity of the peptide analogue as compared with the wild type (18). Both antigen minigenes contained a start and a stop codon. Preparation of concentrated stocks of third-generation lentivectors was performed as described $(8,19)$.

PCR detection of integrated virus. PCR detection of lentivector integration into tissue was performed on genomic DNA from whole lymph nodes and spleen using a seminested approach with GFP-specific primers (forward, 5'-CAAATGGGCGGTAGGCGTGTA-3'; reverse, $5^{\prime}$-TGGGGGTGTTCTGCTGGTAG-3', nested primer, $5^{\prime}$-GGCCACAAGTTCAGCGTGTCC-3').

Histology. Lymph nodes and spleens were embedded in Tissue Tek OCT compound (Miles, Elkhart, Indiana, USA) and snap frozen in 2-methylbutane (Merck, $\mathrm{ZH}$-Dietikon, Switzerland). Cryostat sections $(7 \mu \mathrm{m})$ were either analyzed directly for GFP fluorescence or fixed in 5\% paraformaldehyde for histochemical detection of GFP with rabbit anti-GFP mAb (Aurora, Madison, Wisconsin, USA) for 1 hour at room temperature after rehydration with $\mathrm{PBS}$ and blocking of nonspecific background with $1 \%$ BSA in PBS. Likewise, CD11c, B220, and CD11b were detected using biotinconjugated hamster anti-mouse CD11c (HL3, BD Pharmingen, San Jose, California, USA), biotin-conjugated rat anti-mouse B220 (RA3-6B2, Caltag Laboratories Inc., Burlingame, California, USA), or biotinconjugated rat anti-mouse CD11b (M1/70, BD Pharmingen). As secondary antibody for GFP detection, a goat-anti rabbit IgG conjugated with Alexa 488 was used (Molecular Probes, Juro AG, Lucerne, Switzerland). CD11c, CD11b, and B220 were stained using Streptavidin Cy3 (Jackson ImmunoResearch; Milan Analytica, La Roche, Switzerland). After washing in PBS, stained sections were mounted with 1,4diazabicyclo-(2.2.2)-octane (DABCO) mounting media (Sigma-Aldrich, Buchs, Switzerland).

Synthetic peptides and CPG ODNs. Peptides were synthesized by solid-phase chemistry on a multiple peptide synthesizer (Applied Biosystems, Foster City, California, USA). Peptides were over 90\% pure (by analytical HPLC). Lyophilized peptides were diluted in DMSO and stored at $-20^{\circ} \mathrm{C}$. Synthetic CpG ODN 1826 (TCCATGACGTTCCTGACGTT) optimized for mouse vaccination (Coley Pharmaceutical Group Inc., Wellesley, Massachusetts, USA) was dissolved in sterile PBS.

Flow cytometry. Flow cytometry was performed on a FACSCalibur using Cellquest software (Becton Dickinson, San Jose, California, USA). Peripheral blood lymphocytes (PBLs) obtained by tail bleedings were incubated with phycoerythrin-conjugated $\mathrm{H} 2-\mathrm{K}^{\mathrm{d}} / \mathrm{Cw} 3_{194-203}$ or HLA-A*0201/ELA $26-35$ tetramers (prepared in our laboratory) for 30 minutes at $20^{\circ} \mathrm{C}$ and then washed before incubation with Cychrome-conjugated antiCD8 mAb 53-6.7 (eBioscience, San Diego, California, USA), FITC-conjugated anti-V $\beta 10$ (B21.5), and APCconjugated anti-CD62L (Mel-14, prepared in our laboratory) for 20 minutes at $4^{\circ} \mathrm{C}$. Erythrocytes were lysed using the FACS Lysing solution (Becton Dickinson).

Transduction of bone marrow-derived DCs. Bone marrow-derived DCs (BMDCs) cultured in the presence of GM-CSF (20) were transduced at day 5 of culture by adding concentrated virus (MOI, 100; vector concentration, $5 \times 10^{4}$ expression-forming units [EFUs] per microliter) to the culture medium as described (8). Three days later, the cells were extensively washed and administered to mice.

Vaccination. Peptide vaccination was carried out by subcutaneously injecting $50 \mu \mathrm{g}$ of peptide and $50 \mu \mathrm{g}$ of CpG ODNs emulsified in $50 \mu$ l of incomplete Freund's adjuvant (IFA) and $50 \mu \mathrm{l}$ of PBS into the base of the tail. Recombinant lentivectors were administered by subcutaneously injecting $2 \times 10^{7}$ EFUs in $100 \mu$ of PBS either into the footpad or into the base of the tail. DC-based vaccination was carried out by administering $5 \times 10^{5}$ DCs containing 25-30\% lentivector-transduced or Cw3transgenic DCs into each hind footpad of mice.

CTL assays. In vivo CTL assays were performed as described previously (21). Briefly, splenocytes from syngeneic mice were pulsed or left nonpulsed with Cw3 antigenic peptide (RYLKNGKETL). The pulsed fraction was then labeled in PBS containing CFSE at $0.6 \mu \mathrm{M}$, and the nonpulsed fraction was labeled in 0.04 $\mu \mathrm{M}$ CFSE. Both fractions were adjusted to similar cell content (50:50 ratio), pooled, and injected into the tail vein at $10^{7}$ cells per mouse on day 8 after vaccination with Cw3 cDNA lentivector. Twelve hours later, mice were bled, and the disappearance of peptide-pulsed cells was determined by FACS analysis. By comparing the ratio of the pulsed (high fluorescence intensity) to the nonpulsed fraction (low fluorescence intensity), the percentage of specific killing was established according to the following equation: 100 - ([percentage pulsed $\left.] \times[\text { percentage nonpulsed }]^{-1} \times 100\right)$.

\section{Results}

Targeting of DCs in situ by lentivector. Viruses can be potent inducers of $\mathrm{T}$ cell immunity but also dispose of many ways to avoid immune recognition. This has to be taken into account when evaluating the potential of a 
recombinant virus for the purpose of vaccination. As $T$ cell responses critically depend on the presentation of viral antigens by the MHCs of professional APCs (22, 23), the tropism of a recombinant virus may determine its immunogenicity. The lentivector used in this study was pseudotyped with vesicular stomatitis virus glycoprotein and thus is taken up through the normal endocytotic pathways. Therefore, it is able to transduce a wide variety of cells in vitro, including DCs $(8,9)$.

We analyzed the tissue distribution and the target cells after administration of lentivector vaccine into one footpad of mice by a sensitive PCR assay and by immunohistochemical analysis of lymph nodes and spleen. For PCR analysis, genomic DNA was isolated from the site of injection, the draining (popliteal) lymph node, the spleen, and - as controls - the mandibular lymph nodes and the tip of the tail. Amplification of GFP-specific sequences included in the lentivector vaccine was detected, apart from in the injection site, in only the draining popliteal lymph node and the spleen (Figure 1a).

Monitoring GFP fluorescence on frozen sections of total lymph nodes revealed the presence of transduced cells in the cortex of the draining lymph node but not in lymph nodes of nontreated animals or in the contralateral lymph node of lentivector-treated mice (Figure 1b, upper panels). This fluorescence was found 2-3 days after administration of lentivector. Sections prepared 10 days after lentivector administration did not show any GFP fluorescence (data not shown). The observation that integrated vector persisted at later time points, as revealed by PCR, might be due to the greater sensitivity of this assay.

To identify which cell types were transduced in vivo by lentivector, we combined immunofluorescence analysis of GFP expression with markers specific for DCs, macrophages, and B cells. Doublepositive cells were observed with an antibody against the DC marker CD11c (Figure 1b, lower panels) but not with antibodies to either the $\mathrm{B}$ cell marker CD45R/B220 or the macrophage marker CD11b (Figure $1 \mathrm{~b}$ ) in any of the sections analyzed $(n=30)$. Although the

\section{Figure 1}

majority of GFP-positive cells were CD11c positive, about $10 \%$ of GFP-positive cells were negative for all of the markers tested.

Induction of CTL responses upon lentivector administration. We then tried to elucidate whether the observed in vivo targeting of DCs by lentivector vaccine correlated with efficient induction of CTL responses. To that end, we vaccinated mice with a lentivector bearing a CTLdefined antigen derived from human HLA-Cw3 (referred to throughout the text as $\mathrm{Cw}_{1} 3_{194-203}$ ). The ensuing response was characterized by monitoring the $\mathrm{CD}^{+} \mathrm{T}$ cell response specific to $\mathrm{H}-2 \mathrm{~K}^{\mathrm{d}}$-restricted
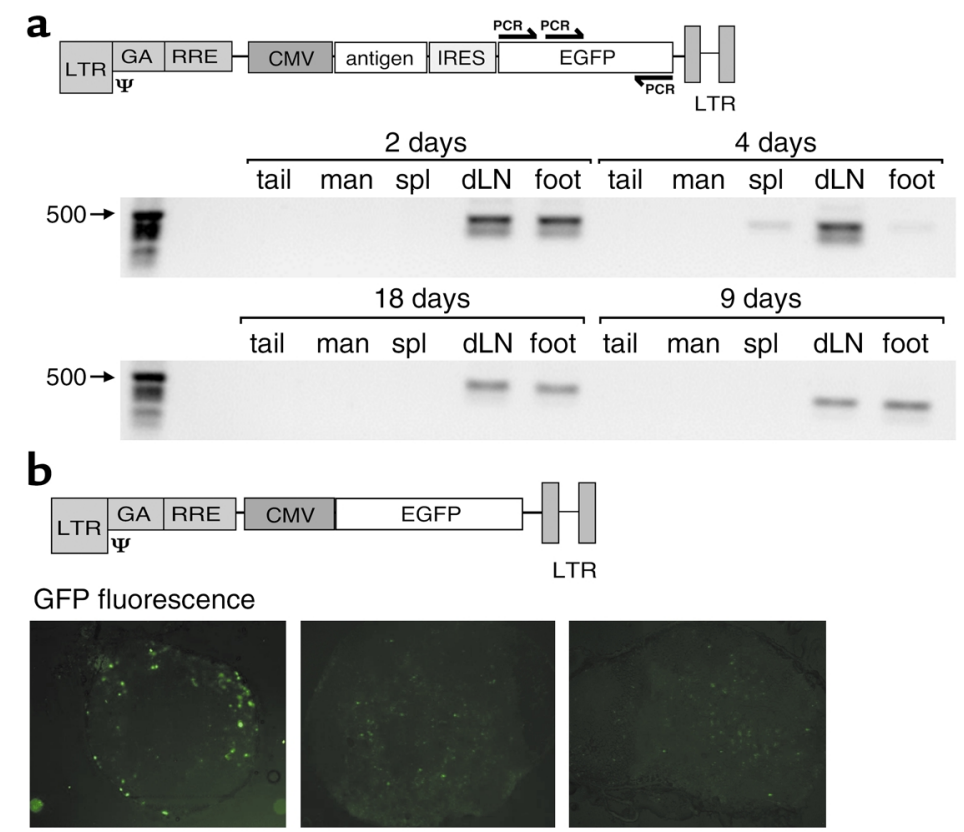

dLN, Iv GFP

Co-staining

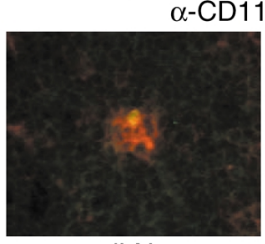

dLN

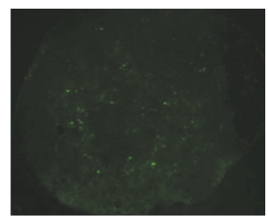

Contra LN, Iv GFP

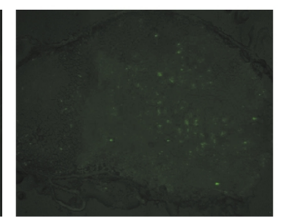

Nontreated LN

$\alpha-B 220 / \alpha-G F P$

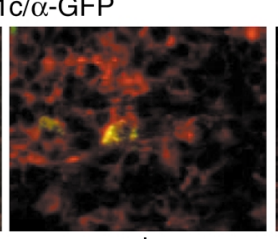

$\mathrm{spl}$

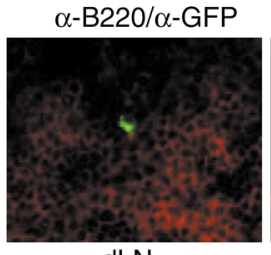

dLN
$\alpha-\mathrm{CD} 11 \mathrm{~b} / \alpha-\mathrm{GFP}$

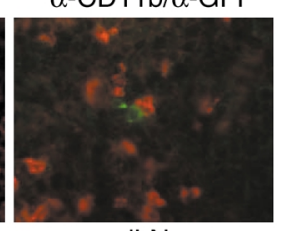

$\mathrm{dLN}$

Tissue distribution of lentivector obtained after in vivo administration into footpads of mice. (a) Detection of integrated lentivector at the site of injection (foot), the draining lymph node, and the spleen but not in the mandibular lymph node and the tip of the tail. Vector integration was detected by amplification of vector-derived GFP sequence with specific primers (arrows) using seminested PCR. dLN, draining lymph node; spl, spleen; man, mandibular lymph node. (b) Histological analysis of GFP expression after administration of lentivector into one hind footpad. The upper panel shows GFP fluorescence of frozen sections of popliteal lymph nodes 2.5 days after injection of CMV-GFP lentivector (IvGFP) or PBS (nontreated) into the footpad. The contralateral popliteal lymph node (contra) of a treated mouse is also shown. Magnification, $\times 100$. The lower panel shows double immunofluorescence analysis of draining lymph nodes and spleen 2.5 days after injection of CMV-GFP lentivector using anti-GFP antibodies (green) counterstained with anti-CD11c, anti-CD11b, and anti-B220 antibodies (red). Magnification, $\times 400$. The use of a CMV-GFP lentivector for immunofluorescence was necessary, because with the bicistronic vaccine constructs (antigen-IRES-GFP), GFP fluorescence was too low to be picked up in histology. For PCR analysis of the GFP sequence, the actual vaccine vector was used. 
a

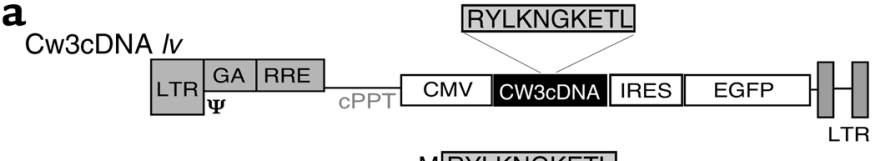

miniCw3 IV M RYLKNGKETL

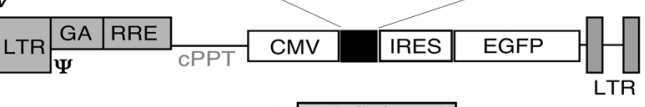

miniMelan-A /V

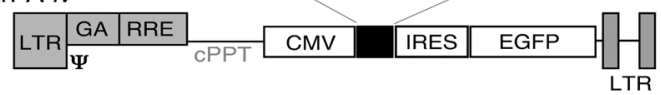

b

Cw3cDNA $/ v$
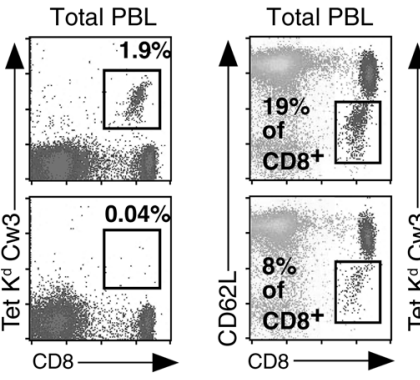

Activated CD8 ${ }^{+}$Total CD8 ${ }^{+}$

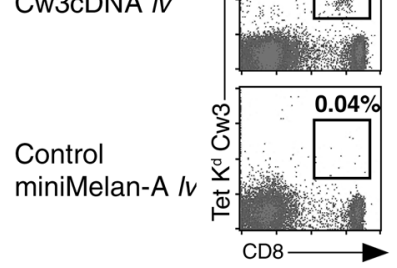

c

Peripheral blood

Input
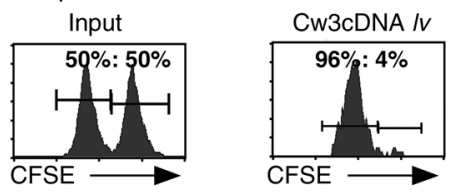

$7 \% \mathrm{Tet}^{+} / \mathrm{CD}^{+}$

$95 \%$ killing

Spleen

Cw3cDNA /

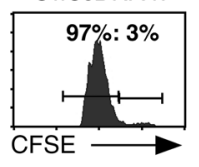

$14 \% \mathrm{Tet}^{+} / \mathrm{CD}^{+}$ 97\% killing

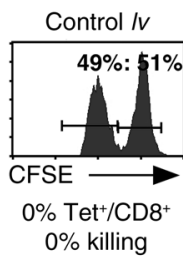

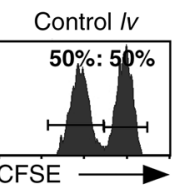

0\% Tet $^{+} / \mathrm{CD}^{+}$

$0 \%$ killing

Liver

Cw3cDNA $/ \mathrm{V}$

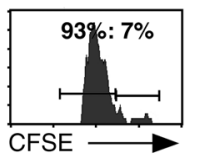

$35 \% \mathrm{Tet}^{+} / \mathrm{CD}^{+}$

93\% killing
Untreated

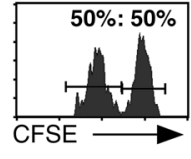

$0 \%$ killing

Control IV

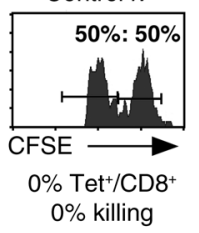

0\% Tet $^{+} / \mathrm{CD}^{+}$
Figure 2

Direct administration of lentivector results in the induction of Cw3 CTL responses. (a) Schematic representation of the constructs used to generate lentiviral vectors. (b) Phenotypic characterization of peak response (day 9). PBLs were analyzed for the cell surface markers CD8, CD62L (downregulated upon activation), and TCR $\mathrm{V} \beta 10$. The $T$ cell receptor specificity was examined using $\mathrm{H}-2 \mathrm{~K}^{\mathrm{d}} / \mathrm{Cw} 3$ tetramers in combination with V $\beta 10$ staining. The proportion of Cw3-specific cells among total PBLs obtained after direct in vivo administration of lentivector is depicted. As a control, an irrelevant (miniMelan-A) lentivector was administered. This is followed by a detailed analysis of the immune response, featuring the proportion of activated $\mathrm{CD}^{+}$cells, the proportion of Cw3-specific cells in the activated compartment, and the proportion of Cw3-specific cells among total CD8 ${ }^{+}$cells. (c) CTL assay showing the in vivo elimination of target cells transferred to vaccinated mice. Syngeneic splenocytes, pulsed with Cw3 antigenic peptide (RYLKNGKETL) and labeled with CFSE at high concentration, were transferred to vaccinated mice 1 day before peak response along with the same number of nonpulsed splenocytes labeled with CFSE at a lower concentration. Twelve hours later, the disappearance of peptide-pulsed cells was determined by FACS analysis in PBL, spleen, and liver. By a comparison of the ratio of pulsed to nonpulsed cells, the percentage of specific killing was calculated. Control $I v$, lentivector expressing an irrelevant CTL epitope; miniMelan-A /v, ELA $26-35$ minigene lentivector.
$\mathrm{Cw} 3_{194-203}$ in PBLs of vaccinated mice by staining with $\mathrm{H}-2 \mathrm{~K}^{\mathrm{d}} \mathrm{Cw} 3_{194-203}$ tetramers $(17,24)$. In combination with tetramer staining, we used antibodies against the $\mathrm{V} \beta 10 \mathrm{~T}$ cell receptor segment, since the majority of $\mathrm{Cw} 3$-specific cells are $\mathrm{V} \beta 10^{+}(25)$. A lentivector bearing the cDNA of HLA-Cw3 (Cw3 cDNA $l v$ ) (Figure 2a) was administered by injecting $2.5 \times 10^{7}$ EFUs into the hind footpads of mice. As a negative control, the same amount of a lentivector expressing an irrelevant antigen (Melan-A) was used (Figure 2a).

The vaccines were well tolerated, and a swelling at the site of infection was usually resolved after 1 day. Administration of $\mathrm{Cw} 3 \mathrm{cDNA}$ lentivector resulted in the induction of a massive $\mathrm{T}$ cell response (Figure $2 \mathrm{~b}$ ), peaking at day 9 and characterized by the presence of $10-40 \%$ antigen-specific cells $\left(\mathrm{H}-2 \mathrm{~K}^{\mathrm{d}} \mathrm{Cw} 3_{194-203}\right.$ tetramer-positive) among total $\mathrm{CD}^{+}$cells (Figure $2 \mathrm{~b}$ ). In comparison with the irrelevant lentivector control, we also observed a large increase in the proportion of activated $\mathrm{CD}^{+}$cells $\left(\mathrm{CD} 62 \mathrm{~L}^{-} / \mathrm{CD}^{+}\right)$(Figure 2b).
In order to test the functional capacity of the induced antigen-specific $\mathrm{CD}^{+}$cells, we performed in vivo CTL assays using as targets syngeneic spleen cells pulsed with antigenic peptide that were transferred to vaccinated mice (21). Before transfer, the pulsed cells were labeled with CFSE, allowing us to monitor their fate in vivo by FACS analysis in tissue such as blood, spleen, and liver. As an internal control, the same number of nonpulsed splenocytes was labeled with a lower intensity of CFSE and coinjected with the pulsed cells. The shift in the ratios of the two CFSE-positive populations provoked by the in vivo elimination of pulsed cells allowed us to calculate the percentage of specific killing that had occurred. Transfer of pulsed and nonpulsed target cells into vaccinated mice was performed 1 day before the peak of the $T$ cell response. Analysis of PBLs, splenocytes, and liver lymphocytes was carried out 12 hours after transfer, and the proportion of $\mathrm{H}-2 \mathrm{~K}^{\mathrm{d}} \mathrm{Cw} 3_{194-203}$ tetramer ${ }^{+} / \mathrm{CD}^{+}$cells, as well as the shift in the ratios of the two CFSE-labeled populations, was determined by FACS analysis. 
As demonstrated in Figure 2c, vaccination with Cw3 cDNA lentivector resulted in an almost complete (over 95\%) elimination of the pulsed, CFSE ${ }^{\text {high }}$ population. In marked contrast, vaccination with a lentivector bearing an irrelevant CTL-defined antigen did not result in significant killing. Moreover, the observed killing activity induced by vaccination with $\mathrm{Cw} 3 \mathrm{cDNA}$ lentivector was systemic, as was demonstrated by the simultaneous and almost complete disappearance of pulsed targets in spleen and liver of vaccinated mice.

Comparative efficiency of direct administration of lentivector with transfer of transduced DCs or peptide/CpG/adjuvant vaccination. Having established that administration of lentivector bearing a $\mathrm{T}$ cell antigen resulted in strong CTL responses, we next wanted to compare its potency with other currently used vaccine protocols. Among these, administration of ex vivo-transduced DCs has set the standards in experimental $\mathrm{T}$ cell vaccination. For ex vivo DC transduction, the same lentivector bearing the cDNA of HLA-Cw3 (Cw3 cDNA $l v$ ) was used as for direct in vivo administration. As described previously (8), DC-based vaccination was performed by injecting transduced BMDCs into the hind footpads of mice (8). As a control for the influence of the viral vector on immune responses, we transferred BMDCs of $\mathrm{Cw} 3$ transgenic mice.

Kinetic analysis of the response (Figure $3 \mathrm{a}$ ) revealed that with all three vaccines, the peak of response was reached around day 9 . Subsequently, the proportion of Cw3-specific cells diminished rapidly to reach near preimmune levels by day 50 . Comparison of the proportion of antigen-specific $\mathrm{CD}^{+} \mathrm{T}$ cells at peak response with the different vaccination approaches demonstrated that direct administration of lentivector resulted, on average, in a higher amplitude of the response (Figure $3 \mathrm{~b}$ ), with maximal responses of up to $42 \%$ of antigen-specific cells among total $\mathrm{CD}^{+} \mathrm{T}$ cells.

A further step in the assessment of the lentivector vaccine was its comparison with another cell-free vaccine: peptide/CPG/adjuvant vaccination. We recently demonstrated that vaccination using synthetic antigenic peptide in combination with CPG ODNs emulsified in IFA was able to induce relatively potent $\mathrm{T}$ cell responses (26). The comparison of lentivector vaccine with $\mathrm{Cw} 3_{194-203}$ peptide/CpG/IFA was done using a lentivector bearing a minigene that encoded the HLA-Cw3 $\left(\mathrm{Cw} 3_{194-203}\right)$

\section{Figure 3}

Comparative analysis of the $\mathrm{CD}^{+} \mathrm{T}$ cell response as induced by lentivector administration, DC-based vaccination, and peptide/CpG/adjuvant vaccination. (a) Comparative vaccination with transgenic DCs, transduced DCs, and direct in vivo administration of lentivector. Mice were vaccinated and PBLs were analyzed by FACS analysis as described. Evolution of the Cw3-specific population over time is shown. All graphs show individual mice that were representative of at least seven mice used for each condition in at least three independent experiments. (b) Proportion of Cw3-specific cells in the $\mathrm{CD}^{+}$compartment as determined at peak response with all vaccination conditions plus preimmune levels (dashed lines and $\mathrm{x}=$ average, $n=$ number of mice tested). (c) Comparative vaccination with $\mathrm{Cw} 3$ minigene lentivector and Cw3 peptide/ $\mathrm{CpG} / \mathrm{IFA}$ vaccination. Shown are time courses of the proportion of activated $C D 8^{+}$cells and the proportion of Cw3-specific cells within the activated population followed by the proportion of Cw3-specific cells in the $\mathrm{CD}^{+}$population. (d) Proportion of Cw3specific cells in the $\mathrm{CD} 8^{+}$compartment as determined at peak response (day 9 for lentivector vaccination and day 7 for peptide-adjuvant vaccination) of all mice tested (dashed lines and $\mathrm{x}=$ average, $n=$ number of mice tested).
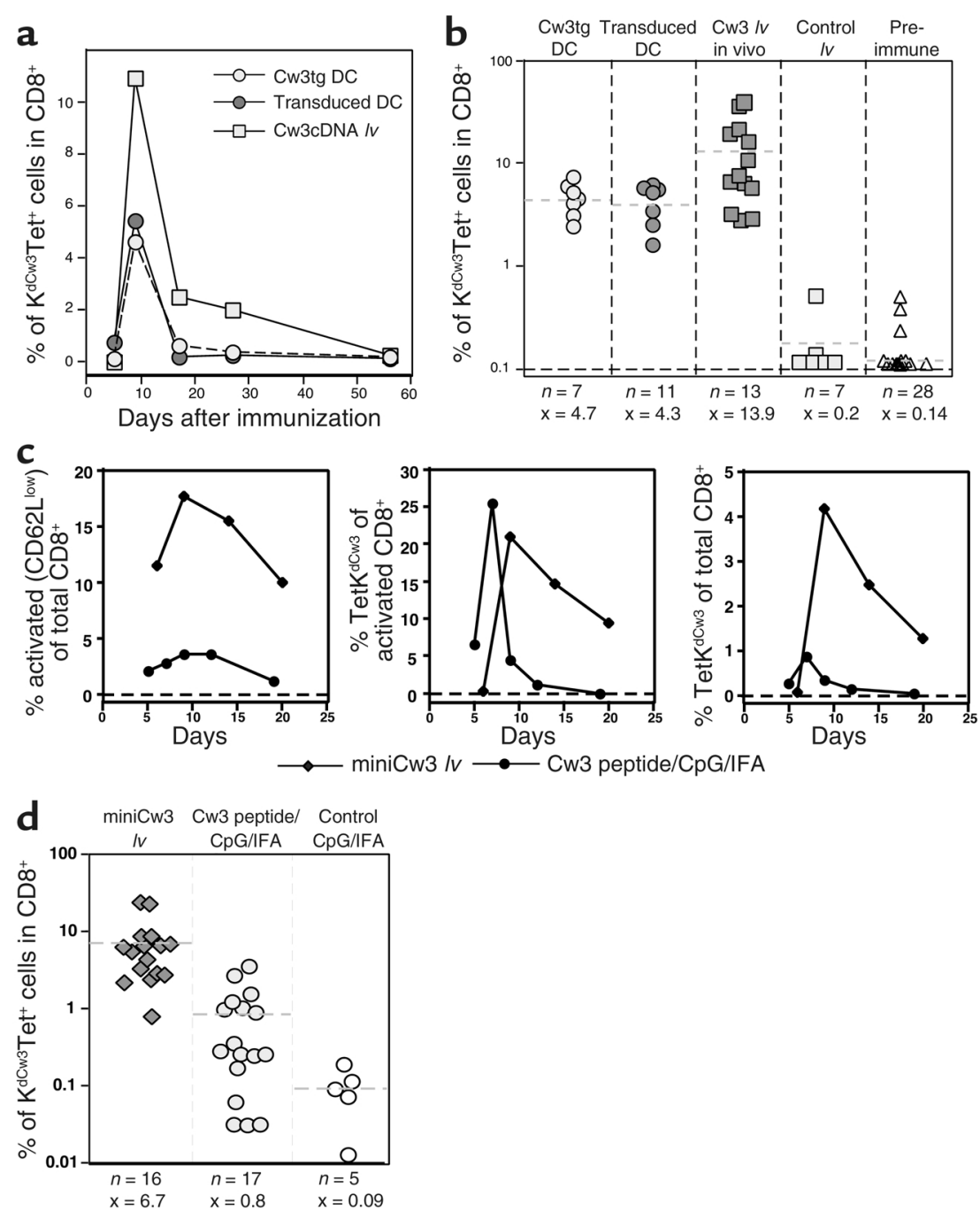

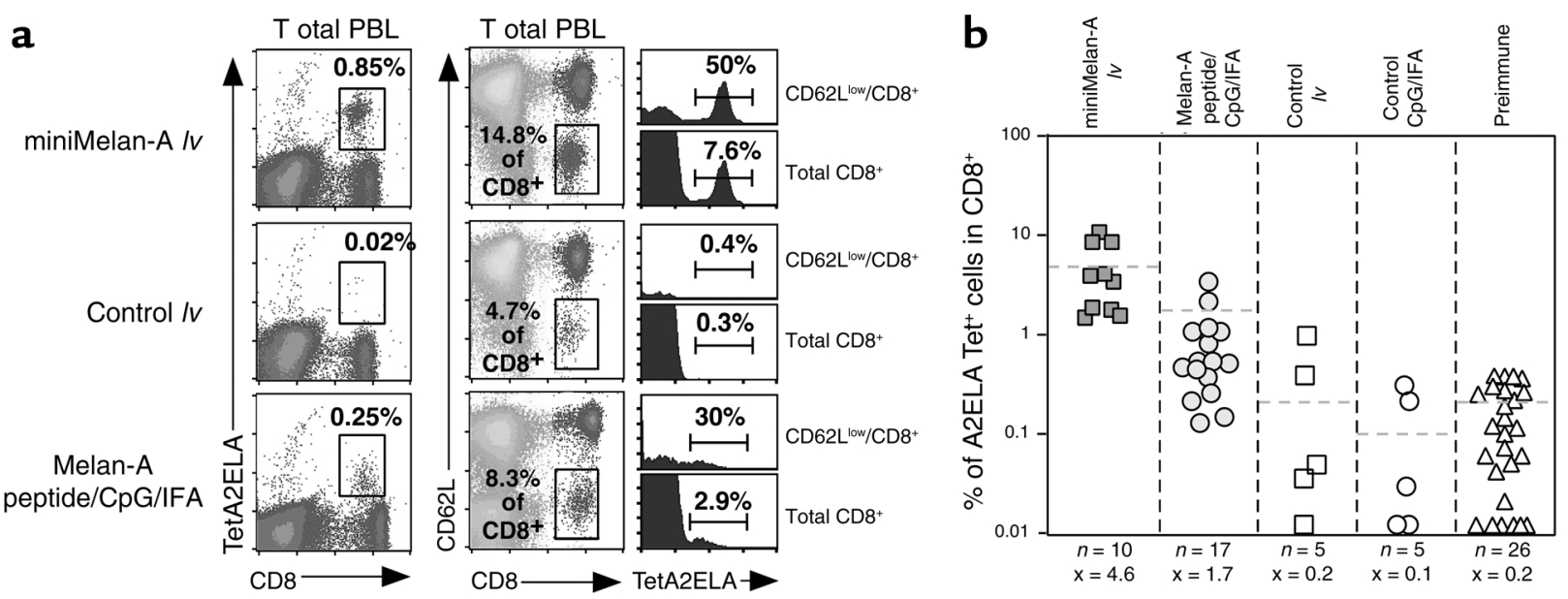

\begin{abstract}
Figure 4
Induction of a Melan-A-specific CD8 ${ }^{+}$T cell response in HLA- $A^{*} 0201 / K^{b}$ transgenic mice vaccinated by administration of lentivector encoding the Melan-A CTL epitope ELA $26-35$. As controls, a lentivector expressing an irrelevant CTL epitope and the administration of ELA $26-35$ pep- $^{-}$ tide in combination with CPG and IFA were used. (a) Phenotypic characterization of the peak of immune response (day 14 for lentivector vaccination and day 7 for peptide/CpG/adjuvant vaccination) of individual mice. Melan-A-specific cells among total PBLs are shown in the first row of panels; the percentage of tetramer-positive cells is indicated. The proportion of activated CD $8^{+}$cells and the percentages of Melan-A-specific cells contained therein and in total CD8 ${ }^{+}$cells are displayed in histograms. (b) Proportion of Melan-A-specific CD8 ${ }^{+}$cells at peak response of all individual mice tested. (Dashed lines and $\mathrm{x}=$ average, $n=$ number of mice tested per condition).
\end{abstract}

derived peptide. Both vaccines were administered subcutaneously in the base of the tail because of the relatively high volume of the peptide-adjuvant emulsion. The immune response induced by lentivector or peptide/CpG/adjuvant vaccination was evaluated by monitoring the evolution of parameters such as activation of $\mathrm{CD}^{+}$cells and the proportion of $\mathrm{Cw} 3$-specific cells among activated and total $\mathrm{CD}^{+}$cells (Figure $3 \mathrm{c}$ ). The most obvious difference between the two vaccines is the 10 -fold higher level of activated $\mathrm{CD}^{+}$cells induced by the lentivector vaccine. This results in turn in a higher proportion of $\mathrm{Cw} 3$-specific cells among total $\mathrm{CD}^{+}$cells. Another difference is that $100 \%$ of the mice treated with lentivector vaccine mounted a $\mathrm{Cw} 3$-specific response, whereas peptide/CpG/adjuvant vaccination achieved a success rate of only $70 \%$ (Figure $3 \mathrm{~d}$ ).

Inducing Melan- $A-$ specific $C D 8^{+} T$ cell responses by direct lentivector administration. The efficacy of direct in vivo administration of lentivector as demonstrated in the Cw3 model was then evaluated in a more clinically relevant model system involving a modified CTLdefined antigen derived from the human melanomaassociated differentiation antigen Melan-A/MART-1 $(27,28)$. This HLA-A*0201-restricted Melan-A antigenic peptide (referred to throughout the text as ELA $_{26-35}$ ) was expressed in lentivector vaccines as a minigene (Figure 1a). Vaccination and characterization of the ensuing immune response was then carried out in HLA-A*0201/ $\mathrm{K}^{\mathrm{b}}$ transgenic mice (A2/ $\mathrm{K}^{\mathrm{b}}$ mice). In addition to endogenous MHC class I molecules, these mice express a chimeric human/ mouse MHC I molecule composed of the $\alpha 1$ and $\alpha 2$ domains of HLA-A2.1 and the $\alpha 3$, transmembrane, and cytoplasmic domains of $\mathrm{H}-2 \mathrm{~K}^{\mathrm{b}}(14)$. The murine elements of the chimeric class I molecule are required for efficient interaction with mouse $\beta 2$-microglobulin and the CD8 coreceptor.

With direct administration of $\mathrm{ELA}_{26-35}$ minigene lentivector, we observed a peak of the response at day 14 , whereas with peptide/CpG/IFA vaccination the peak was reached at day 7 , similar to the situation in the $\mathrm{Cw} 3$ antigen model. A representative example of these peak responses is shown in Figure 4a. Using HLA-A2/ELA $26-35$ tetramers to detect Melan-A-specific $\mathrm{CD}^{+} \mathrm{T}$ cells, we observed a strong, specific response in mice immunized with $\mathrm{ELA}_{26-35}$ minigene lentivector but not in those vaccinated with an irrelevant vector. A comparison of this response to that obtained with peptide/CpG/IFA vaccination shows that ELA $_{26-35}$ minigene lentivector induces more than twofold higher levels of $\mathrm{ELA}_{26-35}$-specific cells among total $\mathrm{CD}^{+}$ cells (Figure 4, $a$ and $b$ ) and more than threefold higher levels among total PBLs (Figure 4a). As seen with the $\mathrm{Cw} 3$ model, this is a direct consequence of the higher levels of $\mathrm{CD}^{+} \mathrm{T}$ cell activation induced by lentivector and the higher proportion of ELA $_{26-35}$-specific cells contained in the activated population.

Recall responses to lentivector vaccination. After the characterization of the strong and sustained primary responses induced by $\mathrm{ELA}_{26-35}$ minigene lentivector, we next evaluated whether a secondary challenge with the same vector would be effective. At the same time, we tested whether antivector immunity had been established during primary immunization. To that end, we performed recall immunizations with the same dose of $\mathrm{ELA}_{26-35}$ minigene lentivector 60 days after primary vaccination in mice primed with either $\mathrm{ELA}_{26-35}$ minigene lentivector or with an irrelevant lentivector not bearing the $\mathrm{ELA}_{26-35} \mathrm{CTL}-$ defined antigen. 


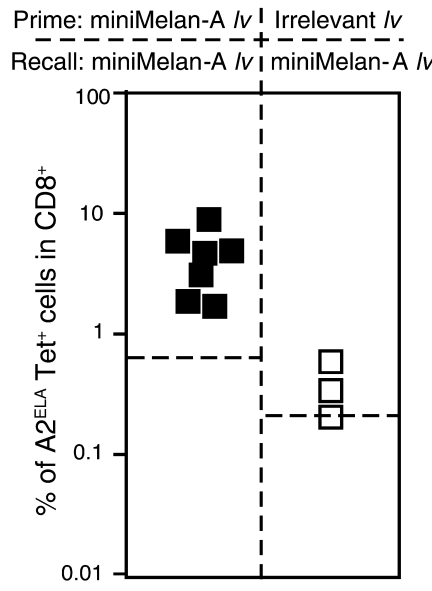

Figure 5

Recall immunizations with miniMelan-A lentivector 60 days after primary immunization with either miniMelan-A lentivector or an irrelevant lentivector. Indicated is the proportion of Melan-A-specific $\mathrm{CD} 8^{+}$cells obtained at the peak of recall response of all individual mice tested (dashed lines indicate the average levels of ELA ${ }_{26-35}$-specific cells present before recall immunization).

Mice that had been primed with an irrelevant lentivirus were unable to mount a significant $\mathrm{ELA}_{26-35^{-}}$ specific $\mathrm{T}$ cell response (Figure 5). This indicated that primary immunization with lentivector resulted in the induction of antivector immunity. However, ELA $26-35$ minigene lentivector-primed mice responded with similar kinetics and amplitude as seen during the primary response (Figure 5 and data not shown).

Dependence of lentivector-induced CTL responses on $\mathrm{CD}^{+}$ $T$ cell help. Most $\mathrm{CD}^{+} \mathrm{T}$ cell responses are dependent on help mediated by antigen-specific $\mathrm{CD}^{+} \mathrm{T}$ cells (29). To address the role of CD4 T cells in CTL responses induced by lentivector, we vaccinated mice that were depleted or not of $\mathrm{CD} 4^{+} \mathrm{T}$ cells. Depletion was carried out by injecting the anti-CD4 mAb GK 1.5 (30) 2 days before vaccine inoculation. This resulted in less than $2 \%$ remaining $\mathrm{CD}^{+} \mathrm{T}$ cells by the day of vaccine inoculation (data not shown). Helper dependency was evaluated both in the Cw3 and the Melan- $\mathrm{A} / \mathrm{A}_{2} / \mathrm{K}^{\mathrm{b}}$ antigen models.

Although vaccination with $\mathrm{Cw} 3 \mathrm{cDNA}$ lentivector was only slightly affected by $\mathrm{CD} 4^{+} \mathrm{T}$ cell depletion, administration of $\mathrm{ELA}_{26-35}$ minigene lentivector to CD4-depleted mice resulted in dramatically decreased proportions of antigen-specific $T$ cells (Figure 6).

\section{Discussion}

Currently, there is no vaccine available that is able to cure cancer. There is, however, evidence that antitumor vaccination can induce specific antitumor $T$ cell responses and even tumor regression $(2,3,31)$. In the overwhelming majority of cases, these regressions were only transient, suggesting that the $\mathrm{T}$ cell responses induced might not have been strong and sustained enough (32). On the basis of these observations, the possibility exists that an optimized vaccination protocol may lead to stronger and curative antitumor $\mathrm{T}$ cell responses.

Toward this end, we evaluated lentiviral vector as a new candidate $T$ cell vaccine. Third-generation lentivector was chosen because of its advanced safety profile, allowing its administration in vivo, and because of the presumed absence of pre-existing antivector immunity in humans. To characterize the properties of lentivector vaccine, we studied its transduced targets and its ability to induce CTL responses in vivo.

In a recent report, the administration of lentivector into the tail vein of mice resulted in transduction in both the liver and the spleen. Within these organs, and particularly in the spleen, the cellular targets of lentivector included a prominent proportion of cells that displayed some markers characteristic of professional APCs (33). However, no clear evidence for the transduction of bona fide DCs was provided.

In our study, lentivector was injected subcutaneously into the footpads, and integrated vector was found mainly at the site of injection, the draining lymph node, and the spleen. We were further able to demonstrate that most transduced cells in the lymphoid organs were CD $11 c^{+}$DCs. The presence of transduced DCs in the draining lymph node and the spleen could be the result of transduction of resident DCs in situ or of migration of DCs that had been transduced at the site of injection. In the latter scenario, lentivector-induced maturation would then result in the migration of DCs to the draining lymph node where, upon presentation of the antigen, they would be able to prime naive $T$ cells. In this context, the ability of lentivector to transduce nondividing cells (34) may be crucial for its efficacy as a vaccine,

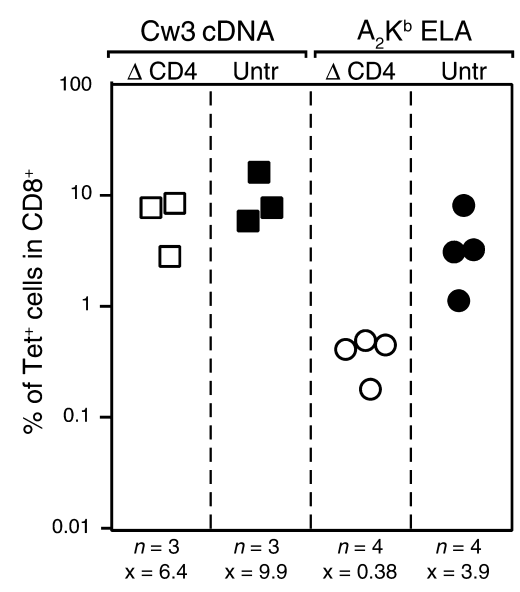

\section{Figure 6}

Influence of $\mathrm{CD}^{+} \mathrm{T}$ cells on induction of $\mathrm{CD} 8^{+} \mathrm{T}$ cell responses by lentivector vaccine. Mice depleted of $\mathrm{CD}^{+} \mathrm{T}$ cells or nondepleted were vaccinated with lentivector vaccine. Indicated are the T cell responses at peak response of groups of mice in the $\mathrm{Cw} 3$ model vaccinated with $\mathrm{Cw} 3 \mathrm{cDNA} / v$ and the Melan- $\mathrm{A} / \mathrm{A}_{2} / \mathrm{K}^{\mathrm{b}}$ antigen model vaccinated with $\mathrm{ELA}_{26-35}$ minigene lentivector $(\mathrm{x}=$ average, $n=$ number of mice tested per condition). $\triangle \mathrm{CD} 4$, mice depleted of $\mathrm{CD} 4^{+} \mathrm{T}$ cells; untr, nondepleted mice. 
since immature DCs are thought to be nondividing (35). The observation that the majority of transduced cells found in the spleen and draining lymph nodes are DCs further supports this theory, since it is unlikely that free viral particles reaching the lymph node or spleen would exclusively infect DCs.

Our observations support the idea that $\mathrm{T}$ cell responses obtained after lentivector administration are due to direct priming of CTL precursors in situ. In view of the strength of the response as compared with the relative paucity of transduced DCs, an additional involvement of cross-priming by antigen taken up by DCs at the site of injection cannot, however, be excluded.

In order to quantitate the efficiency of lentivector vaccination, we chose the direct monitoring of antigen-specific $\mathrm{T}$ cells by tetramer staining and, in the initial characterization of the $\mathrm{Cw} 3 \mathrm{cDNA}$ lentivector, an in vivo CTL assay. We show that lentivector administration induces high levels of antigen-specific $\mathrm{CD}^{+} \mathrm{T}$ cells that display a high CTL activity in vivo. Moreover, although quantitative differences in the outcome of distinct vaccination protocols are difficult to evaluate, the comparison with two of the major existing approaches, peptide/adjuvant vaccination and transfer of antigen-transduced DCs, clearly demonstrated that direct administration of lentivector induces a highly effective antigen-specific $\mathrm{CD}^{+} \mathrm{T}$ cell response.

Since most antitumor $\mathrm{T}$ cell vaccination protocols rely on a prime boost strategy, we evaluated whether lentivector administration resulted in the induction of antivector immunity. Because of the failure to induce a Melan-A-specific response in mice that had been primed with an irrelevant lentivector, we presume that antivector immunity had been established. This could be a potentially limiting factor for repetitive lentivector immunization that needs to be examined further. Nevertheless, the induction of antivector immunity did not prevent repetitive vaccination from being effective when the Melan-A minigene vector was used for priming.

As a further step in the characterization of the lentiviral vaccine, we addressed the dependency of the $\mathrm{CD}^{+} \mathrm{T}$ cell response on help by $\mathrm{CD} 4^{+} \mathrm{T}$ cells. According to a popular model, $\mathrm{T}$ cell help is thought to augment $\mathrm{CD} 8^{+}$responses indirectly through the activation of DCs $(36-38)$. This implies that the action of $\mathrm{CD}^{+} \mathrm{T}$ cell help could be substituted by any another mechanism that leads to appropriate DC activation, including transduction with a recombinant vector. When assessing helper dependency in the $\mathrm{Cw} 3$ and the Melan- $\mathrm{A} / \mathrm{A}_{2} / \mathrm{K}^{\mathrm{b}}$ antigen models, we found that the two models differ in their dependency on $\mathrm{CD}^{+} \mathrm{T}$ cell help. Whereas with $\mathrm{Cw} 3$ no significant difference in the response was observed whether $\mathrm{CD}^{+} \mathrm{T}$ cells were present or not, vaccination with the $\mathrm{ELA}_{26-35}$ minigene lentivector heavily relied on the presence of $\mathrm{CD}^{+} \mathrm{T}$ cells. In the latter case, it appears that a helper epitope must have been provided either by the GFP marker or the viral particle, since the vector expresses only the CTL antigen of Melan-A.

A possible explanation for the divergence in the dependency on $\mathrm{CD}^{+} \mathrm{T}$ cells could be provided by recent data demonstrating that a high frequency of CTL precursors can compensate for CD4 help $(39,40)$. According to this scenario, the responses seen in the Cw3 model would have been mediated by CD4-independent help, whereas in the Melan- $\mathrm{A} / \mathrm{A} 2 / \mathrm{K}^{\mathrm{b}}$ model with low precursor frequencies (41), the presence of $\mathrm{CD}^{+} \mathrm{T}$ cells is needed to induce a $\mathrm{CD} 8^{+} \mathrm{T}$ cell response. In conclusion, data presented here demonstrate the efficacy of direct in vivo administration of lentiviral vectors for the induction of antigen-specific CTL responses. A decisive factor for this efficacy appears to be the in vivo targeting of DCs. From a practical point of view, our results demonstrate that the time-consuming and costly steps currently used to elicit tumorspecific CTL responses through the transfer of ex vivo-manipulated DCs could be replaced by the much simpler direct in vivo administration of antigenrecombinant lentivectors.

\section{Acknowledgments}

The authors are very grateful to the lab of L. Naldini for providing us with the lentiviral vector system, G. Badic for expert technical assistance with histology, and R. Voyle for critical reading of the manuscript. This work was supported in part by a fellowship from the Gabriella Giorgi-Cavaglieri Foundation (to H.R. MacDonald). L. Chapatte and F. Lévy are supported by a grant from the Swiss National Funds. F. Lévy is partially supported by an investigator award from the Cancer Research Institute.

1.Zitvogel, L., et al. 1996. Therapy of murine tumors with tumor peptide-pulsed dendritic cells: dependence on T cells, B7 costimulation, and T helper cell 1-associated cytokines. J. Exp. Med. 183:87-97.

2. Nestle, F.O., et al. 1998. Vaccination of melanoma patients with peptideor tumor lysate-pulsed dendritic cells. Nat. Med. 4:328-332.

3. Thurner, B., et al. 1999. Vaccination with mage-3A1 peptide-pulsed mature, monocyte-derived dendritic cells expands specific cytotoxic $T$ cells and induces regression of some metastases in advanced stage IV melanoma. J. Exp. Med. 190:1669-1678.

4. Brossart, P., Goldrath, A.W., Butz, E.A., Martin, S., and Bevan, M.J. 1997. Virus-mediated delivery of antigenic epitopes into dendritic cells as a means to induce CTL. J. Immunol. 158:3270-3276.

5. Song, W., et al. 1997. Dendritic cells genetically modified with an adenovirus vector encoding the cDNA for a model antigen induce protective and therapeutic antitumor immunity. J. Exp. Med. 186:1247-1256.

6. Germain, R.N., and Margulies, D.H. 1993. The biochemistry and cell biology of antigen processing and presentation. Annu. Rev. Immunol. 11:403-450.

7. Rosenberg, S.A., et al. 1998. Immunizing patients with metastatic melanoma using recombinant adenoviruses encoding MART-1 or gp100 melanoma antigens. J. Natl. Cancer Inst. 90:1894-1900.

8. Esslinger, C., Romero, P., and MacDonald, H.R. 2002. Efficient transduction of dendritic cells and induction of a T-cell response by thirdgeneration lentivectors. Hum. Gene Ther. 13:1091-1100.

9. Gruber, A., Kan-Mitchell, J., Kuhen, K.L., Mukai, T., and Wong-Staal, F. 2000. Dendritic cells transduced by multiply deleted HIV-1 vectors exhibit normal phenotypes and functions and elicit an HIV-specific cytotoxic T-lymphocyte response in vitro. Blood. 96:1327-1333.

10. Zarei, S., Leuba, F., Arrighi, J.F., Hauser, C., and Piguet, V. 2002. Transduction of dendritic cells by antigen-encoding lentiviral vectors permits antigen processing and MHC class I-dependent presentation. J. Allergy Clin. Immunol. 109:988-994.

11. Salmon, P., et al. 2001. Transduction of CD34+ cells with lentiviral vectors 
enables the production of large quantities of transgene-expressing immature and mature dendritic cells. J. Gene Med. 3:311-320.

12. Zufferey, R., et al. 1998. Self-inactivating lentivirus vector for safe and efficient in vivo gene delivery. J. Virol. 72:9873-9880.

13. Dill, O., Kievits, F., Koch, S., Ivanyi, P., and Hammerling, G.J. 1988. Immunological function of HLA-C antigens in HLA-Cw3 transgenic mice. Proc. Natl. Acad. Sci. U. S. A. 85:5664-5668.

14. Vitiello, A., Marchesini, D., Furze, J., Sherman, L.A., and Chesnut, R.W. 1991. Analysis of the HLA-restricted influenza-specific cytotoxic T lymphocyte response in transgenic mice carrying a chimeric human-mouse class I major histocompatibility complex. J. Exp. Med. 173:1007-1015.

15. Maryanski, J.L., Accolla, R.S., and Jordan, B. 1986. H2-restricted recognition of cloned HLA class I gene products expressed in mouse cells. J. Immunol. 136:4340-4347.

16. Bour, H., Horvath, C., Lurquin, C., Cerottini, J.C., and MacDonald, H.R. 1998. Differential requirement for CD4 help in the development of an antigen-specific CD8+ T cell response depending on the route of immunization. J. Immunol. 160:5522-5529.

17. Maryanski, J.L., Pala, P., Corradin, G., Jordan, B.R., and Cerottini, J.C. 1986. H-2-restricted cytolytic T cells specific for HLA can recognize a synthetic HLA peptide. Nature. 324:578-579.

18. Valmori, D., et al. 1998. Enhanced generation of specific tumor-reactive CTL in vitro by selected Melan-A/MART-1 immunodominant peptide analogues. J. Immunol. 160:1750-1758.

19. Dull, T., et al. 1998. A third-generation lentivirus vector with a conditional packaging system. J. Virol. 72:8463-8471.

20. Inaba, K., et al. 1992. Generation of large numbers of dendritic cells from mouse bone marrow cultures supplemented with granulocyte/macrophage colony-stimulating factor. J. Exp. Med. 176:1693-1702.

21. Barchet, W., et al. 2000. Direct quantitation of rapid elimination of viral antigen-positive lymphocytes by antiviral CD8(+) T cells in vivo. Eur. J. Immunol. 30:1356-1363.

22. Sigal, L.J., Crotty, S., Andino, R., and Rock, K.L. 1999. Cytotoxic T-cell immunity to virus-infected non-haematopoietic cells requires presentation of exogenous antigen. Nature. 398:77-80.

23. Sigal, L.J., and Rock, K.L. 2000. Bone marrow-derived APCs are required for the generation of cytotoxic $\mathrm{T}$ lymphocyte responses to viruses and use transporter associated with antigen presentation (TAP)-dependent and independent pathways of antigen presentation. J. Exp. Med. 192:1143-1150

24. Bour, H., Michielin, O., Bousso, P., Cerottini, J.C., and MacDonald, H.R. 1999. Dramatic influence of $V$ beta gene polymorphism on an antigenspecific CD8+ T cell response in vivo. J. Immunol. 162:4647-4656.

25. MacDonald, H.R., Casanova, J.L., Maryanski, J.L., and Cerottini, J.C. 1993. Oligoclonal expansion of major histocompatibility complex class I-restricted cytolytic $\mathrm{T}$ lymphocytes during a primary immune response in vivo: direct monitoring by flow cytometry and polymerase chain reaction. J. Exp. Med. 177:1487-1492.
26. Miconnet, I., et al. 2002. CpG are efficient adjuvants for specific CTL induction against tumor antigen-derived peptide. J. Immunol. 168:1212-1218.

27. Coulie, P.G., et al. 1994. A new gene coding for a differentiation antigen recognized by autologous cytolytic $\mathrm{T}$ lymphocytes on HLA-A2 melanomas. J. Exp. Med. 180:35-42.

28. Kawakami, Y., et al. 1994. Identification of the immunodominant peptides of the MART-1 human melanoma antigen recognized by the majority of HLA-A2-restricted tumor infiltrating lymphocytes. J. Exp. Med. 180:347-352.

29. Malek, T.R. 2002. T helper cells, IL-2 and the generation of cytotoxic T-cell responses. Trends Immunol. 23:465-467.

30. Buller, R.M., Holmes, K.L., Hugin, A., Frederickson, T.N., and Morse, H.C., III. 1987. Induction of cytotoxic T-cell responses in vivo in the absence of CD4 helper cells. Nature. 328:77-79.

31. Rosenberg, S.A., et al. 1998. Immunologic and therapeutic evaluation of a synthetic peptide vaccine for the treatment of patients with metastatic melanoma. Nat. Med. 4:321-327.

32. Yu, Z., and Restifo, N.P. 2002. Cancer vaccines: progress reveals new complexities. J. Clin. Invest. 110:289-294. doi:10.1172/JCI200216216.

33. VandenDriessche, T., et al. 2002. Lentiviral vectors containing the human immunodeficiency virus type- 1 central polypurine tract can efficiently transduce nondividing hepatocytes and antigen-presenting cells in vivo. Blood. 100:813-822.

34. Follenzi, A., Ailles, L.E., Bakovic, S., Geuna, M., and Naldini, L. 2000. Gene transfer by lentiviral vectors is limited by nuclear translocation and rescued by HIV-1 pol sequences. Nat. Genet. 25:217-222.

35. Matsuno, K., Ezaki, T., Kudo, S., and Uehara, Y. 1996. A life stage of particle-laden rat dendritic cells in vivo: their terminal division, active phagocytosis, and translocation from the liver to the draining lymph. J. Exp. Med. 183:1865-1878.

36. Ridge, J.P., Di Rosa, F., and Matzinger, P. 1998. A conditioned dendritic cell can be a temporal bridge between a CD4+ T-helper and a T-killer cell. Nature. 393:474-478.

37. Bennett, S.R., et al. 1998. Help for cytotoxic-T-cell responses is mediated by CD40 signalling. Nature. 393:478-480.

38. Schoenberger, S.P., Toes, R.E., van der Voort, E.I., Offringa, R., and Melief, C.J. 1998. T-cell help for cytotoxic T lymphocytes is mediated by CD40-CD40L interactions. Nature. 393:480-483.

39. Wang, B., et al. 2001. Multiple paths for activation of naive CD8+ T cells: CD4-independent help. J. Immunol. 167:1283-1289.

40. Mintern, J.D., Davey, G.M., Belz, G.T., Carbone, F.R., and Heath, W.R. 2002. Cutting edge: precursor frequency affects the helper dependence of cytotoxic T cells. J. Immunol. 168:977-980.

41. Firat, H., et al. 2002. Comparative analysis of the CD8(+) T cell repertoires of $\mathrm{H}-2$ class I wild-type/HLA-A2.1 and H-2 class I knockout/HLAA2.1 transgenic mice. Int. Immunol. 14:925-934. 